\title{
Non-brittle rachis 1-A (Btr1-A) gene in di- and hexaploid wheat species
}

\author{
V. Vavilova*, I. Konopatskaia, N.P. Goncharov \\ Institute of Cytology and Genetics, SB RAS, Novosibirsk, Russia
}

DOI 10.18699/ICG-PlantGen2019-65

(c) Autors, 2019

* e-mail: valeriya-vavilova@bionet.nsc.ru

\begin{abstract}
Spike brittleness is one of the key domestication traits in Triticum species. In the recent studies it was found that the Non-brittle rachis 1-A (Btr1-A) gene involved in the regulation of the brittle/non-brittle spike trait. Here we investigated the genetic variability of the Btr1-A gene from 13 accessions of Triticum monococcum L., T. urartu Thum. ex Gandil., T. boeoticum Boiss., T. macha Decapr. et Menabde, T. aestivum ssp. petropavlovskyi (Udacz. et Migusch.) N.P. Gontsch., T. spelta L., T. spelta ssp. yunnanense (King ex S.L. Chen) N.P. Gontsch., T. vavilovii (Thum.) Jakibz. and T. tibetanum Shao. The Btr1-A sequences for T. aestivum ssp. petropavlovskyi and T. vavilovii were obtained for the first time. Hexaploid wheat accessions analyzed were characterized by a 2-bp deletion in the Btr1-A coding region (positions 291-299). The presence of this deletion leads to the formation of a nonfunctional protein (97 instead of 196 amino acids). Additional investigations are required to establish the potential relationship between Btr1-A and other genes that regulate the brittle/non-brittle spike trait in Triticum species.

Key words: wheat; Triticum; spike morphology; brittle rachis; Btr1-A gene.
\end{abstract}

\section{Introduction}

Currently, wheat (Triticum L.) is an important cereal in the agriculture, one of the first that was domesticated (Purugganan, Fuller, 2009). Along with spike shape, threshability and spring growth habit, spike brittleness is one of the key domestication traits (Goncharov, 2012). It has been shown that the mutation of the Non-brittle rachis 1-A (Btr1-A) gene controls the brittle/non-brittle spike trait in Triticum species (Zhao et al., 2019). Pourkheirandish et al. (2018) found that the non-synonymous change at the coding region of $B \operatorname{tr} 1-A$ ( $\mathrm{G}$ to A, A119T) leads to non-brittle rachis formation in the diploid einkorn wheat Triticum monococcum, compared to its wild progenitor Triticum boeoticum with a brittle rachis spike. In cultivated polyploid wheat species, the Btr $1-A$ gene contains a 2-bp deletion in the coding region, forming a premature stop codon and resulting in a non-functional protein (Zhao et al., 2019). Moreover, the transgenic wheat lines created by Zhao et al. (2019) made it possible to establish that the Btr $1-A$ gene was associated with spike density, grain size and grain yield. In the present study we investigated the genetic variability of the $B \operatorname{tr} 1-A$ gene from di- and hexaploid wheat species including four endemics.

\section{Materials and methods}

The germplasm of di- and hexaploid wheat species was grown under standard greenhouse conditions. The brittle/non-brittle spike trait was determined visually. The list of wheat accessions analyzed in this study is presented in Table 1. Total DNA was isolated from $100 \mathrm{mg}$ of leaves using a DNeasy Plant Mini Kit (QIAGEN) according to the manufacturer's protocol. The Btrl-A sequences of T. monococcum and T. boeoticum (MG596311-MG596321) and whole genome sequences (WGS) of Triticum dicoccoides (LSYQ02000006), Triticum aestivum (OETA01178479) (B-genome), T. aestivum (OETA01219489) and Aegilops tauschii (NWVB01000003) (D-genome) were used to design genome-specific primers to amplify the Btr $1-A$ gene from di- and hexaploid wheat species. The primer pair Btr-A1-F/ Btr-A1-R 5'-CGAGCTTGACCT CATGTAAC-3'/ 5'-CTACTGCATCATCAGTCCATC-3' amplifies partial upstream and downstream parts and the coding region of Btr 1-A. PCR was performed in a $20-\mu \mathrm{l}$ volume containing $20 \mathrm{ng}$ of genomic DNA, $10 \mathrm{mM}$ Tris- $\mathrm{HCl}$ ( $\mathrm{pH} 8.9$ ), $1 \mathrm{mM}\left(\mathrm{NH}_{4}\right)_{2} \mathrm{SO}_{4}, 1.5 \mathrm{mM} \mathrm{MgCl}_{2}, 200 \mu \mathrm{M}$ dNTPs, $0.5 \mu \mathrm{M}$ primers, and $0.25 \mathrm{U}$ of Taq DNA polymerase. PCR products were separated by agarose gel electrophoresis and purified using a QIAquick Gel Extraction Kit (QIAGEN). For all wheat accessions purified PCR products were sequenced. Sequencing reactions were performed with $20 \mathrm{ng}$ of the PCR product and an ABI BigDye Terminator Kit on an ABI 3130XL Genetic Analyser (Applied Biosystems) in the SB RAS Genomics Core Facility (http://www.niboch.nsc.ru/doku.php/corefacility). Nucleotide and amino acid sequences were aligned using AliView v. 1.18.1.

\section{Results and discussion}

We obtained the Btr1-A gene sequences for three diploid and five hexaploid wheat species (Table 1). For diploid wheat accessions, the length of the Btr $1-A$ sequences obtained was $902 \mathrm{bp}$. In the case of all hexaploid wheat accessions investigated, the length of the gene sequences was $899 \mathrm{bp}$ (a 1-bp deletion in the upstream and a 2-bp deletion in the coding region of the Btrl-A gene compared with diploids).

Comparative analyses allowed us to determine that the Btrl-A gene sequences of T. monococcum (PI-266844) and T. boeoticum (K-18399) were identical with T. monococcum (MG596319, MG596320) and T. boeoticum (MG596311MG596313, MG596315, MG596317-MG596318), respectively. The T. urartu (Ig-110784) sequence contained an additional substitution at position 125 ( $\mathrm{T}$ to A, L42Q) compared to the data presented by Zhao et al. (2019) (Figure 1). The 2-bp deletion in the Btr1-A coding region of T. spelta and T. spelta ssp. yunnanense (non-brittle rachis), T. tibetanum and 
Table 1

Wheat species used in the study and their phenotypes

\begin{tabular}{lll}
\hline Species & Accession & Phenotype \\
\hline Triticum monococcum L. & $\mathrm{Pl}-266844$ & Non-brittle rachis \\
T. urartu Thum. ex Gandil. & $\mathrm{lg}-110784$ & Brittle rachis \\
T. boeoticum Boiss. & $\mathrm{K}-18399$ & Brittle rachis \\
T. macha Decapr. et Menabde & $\mathrm{K}-31689$ & Brittle rachis* \\
T. macha Decapr. et Menabde & $\mathrm{K}-58671$ & Brittle rachis* \\
T. aestivum ssp. petropavlovskyi (Udacz. et Migusch.) N.P. Gontsch. & $\mathrm{KU} 502$ & Non-brittle rachis \\
T. aestivum ssp. petropavlovskyi (Udacz. et Migusch.) N.P. Gontsch. & $\mathrm{K}-43351$ & Non-brittle rachis \\
T. spelta L. & $\mathrm{K}-53364$ & Non-brittle rachis \\
T. spelta ssp. yunnanense (King ex S.L. Chen) N.P. Gontsch. & KU506 & Non-brittle rachis \\
T. spelta ssp. yunnanense (King ex S.L. Chen) N.P. Gontsch. & KU509 & Non-brittle rachis \\
T. vavilovii (Thum.) Jakibz. & Tri9416 & Non-brittle rachis \\
T. tibetanum Shao & KU510 & Brittle rachis* \\
T. tibetanum Shao & KU515 & Brittle rachis*
\end{tabular}

* the spike is separated from the whole straw, rarely without breaking into spikelets (Dorofeev et al., 1979)

T. urartu (Ig-110784)
T. monococcum (PI-266844)
T. boeoticum (K-18399)
T. macha (K-31689, K-58671)
T. aestivum ssp. petropavlovskyi (KU502, K-43351)
T. spelta (K-53364)
T. spelta ssp. yumnanense (KU506, KU509)
T. tibetamum (KU510, KU515)
T. vavilovii (Tri9416)
T. urartu (Ig-110784)
T. monococcum (PI-266844)
T. boeoticum (K-18399)
T. macha (K-31689, K-58671)
T. aestivum ssp. petropavlovskyi (KU502, K-43351)
T. spelta (K-53364)
T. spelta ssp. yumnamense (KU506, KU509)
T. tibetamum (KU510, KU515)
T. vavilovii (Tri9416)
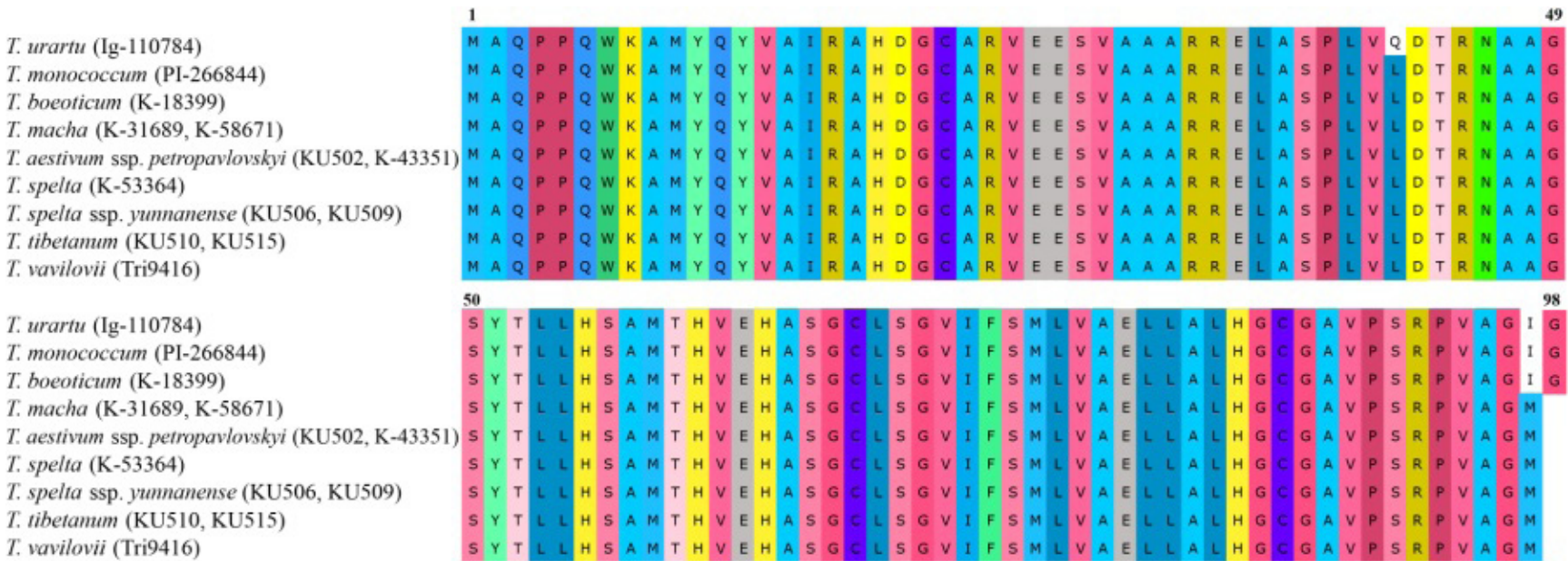

T. urartu (Ig-110784) T. monococcum (PI-266844) T. boeoticum (K-18399)
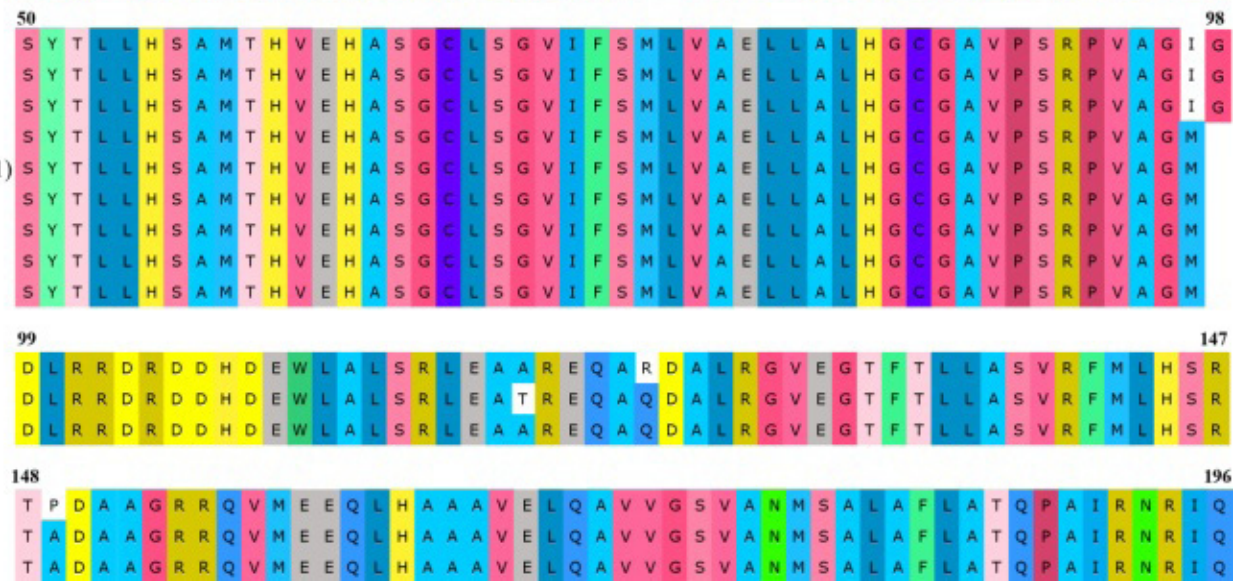

Figure 1. Alignment of the BTR1 protein of the di- and hexaploid wheat accessions analyzed in this study. The length of the BTR1 protein is 196 and 97 amino acids for the diploids and the hexaploids, respectively. 
T. macha (brittle rachis) was previously detected by Zhao et al. (2019). The T. aestivum ssp. petropavlovskyi and T. vavilovii accessions with non-brittle rachis that were investigated in this study also contain that deletion. This deletion formed a premature stop codon and resulted in a nonfunctional protein (Figure 1). The 1-bp deletion at position -97 from the start codon for all hexaploid wheat accessions was detected for the first time in the present study.

\section{Conclusions}

In the present study we investigated the genetic variability of the Btr1-A gene from several Triticum species including four endemics. All hexaploid wheat accessions were characterized by a 2-bp deletion in the Btr $1-A$ coding region, leading to the formation of a nonfunctional protein (97 amino acids instead of 196 in diploids). The Btr $1-A$ sequences for T. aestivum ssp. petropavlovskyi and T. vavilovii were established for the first time in this study. Nevertheless, further investigations are required to understand the potential relationship between the $B \operatorname{tr} l$ and $Q$ genes, regulating spike morphology traits such as spike density, brittleness and grain weight.

\section{References}

Dorofeev V.F., Filatenko A.A., Migushova E.F., Udachin R.A., Jakubtsiner M.M. Pshenitsa (Wheat). In: Dorofeev V.F., Korovina O.N. (Eds.). Cultivated flora of the USSR, vol 1. Leningrad: Kolos, 1979; $348 \mathrm{pp}$.

Goncharov N.P. Comparative genetics of wheats and their related species. Novosibirsk: Geo, 2012; 523 pp.

Pourkheirandish M., Dai F., Sakuma S., Kanamori H., Distelfeld A., Willcox G., Kawahara T., Matsumoto T., Kilian B., Komatsuda T. On the origin of the non-brittle rachis trait of domesticated einkorn wheat. Front Plant Sci. 2018;8:1-10. DOI 10.3389/fpls.2017.02031.

Purugganan M.D., Fuller D.Q. The nature of selection during plant domestication. Nature. 2009;457:843-848. DOI 10.1007/978-94-007$7572-5$ - 18.

Zhao Y., Xie P., Guan P., Wang Y., Li Y., Yu K., Xin M., Hu Z., Yao Y., Ni Z., Sun Q., Xie C., Peng H. Btrl-A induces grain shattering and affects spike morphology and yield-related traits in wheat. Plant Cell Physiol. 2019;60:1342-1353. DOI 10.1093/pcp/pcz050.

Acknowledgements. This study was supported by the Russian Science Foundation (grant No. 16-16-10021-P).

Conflict of interest. The authors declare no conflict of interest. 